\title{
Assistência odontológica e universalização: percepção de gestores municipais
}

\author{
Dental care and universalization: perceptions \\ of public health services managers
}

\footnotetext{
1 Departamento de Odontopediatria Ortodontia e Saúde Coletiva, Faculdade de Odontologia de Bauru, USP Alameda Octávio Pinheiro Brisolla 9-75, Vila Nova Cidade Universitária, 17012-901, Bauru SP. netomita@usp.br
}

\begin{abstract}
This study aimed at qualitatively evaluating the perception of local health managers as to the universalization principle along SUS (Sistema Unico de Saúde) and the access to attention to oral health. Some public health services managers from two cities of the west and mid-west regions of São Paulo State were interviewed. The methodology was based on interviews, utilizing a semi-structured script about access to dental care, main difficulties, and possibilities to confront them. The writings were taped integrally and qualitatively analyzed. The low resolution and restrict access to basic attention in oral health are some of the main problems faced by managers and professionals in the oral health team. The managers presented a perception of scarce possibility of access to dental care along with the public services provided in their cities. The main difficulties at facing the equation demand - effectiveness of dental assistance are discussed.
\end{abstract}

Key words Health policy; Dentistry; Qualitative research
Resumo Este estudo tem por objetivo analisar a percepção de gestores locais de saúde quanto ao acesso à assistência odontológica e o princípio de universalização do Sistema Único de Saúde (SUS). O universo da pesquisa foi constituído por gestores locais de dois municípios das regiões oeste e centro-oeste do Estado de São Paulo. A metodologia baseou-se em entrevista com roteiro semi-estruturado, coleta de depoimentos sobre o acesso da população à assistência odontológica, as principais dificuldades percebidas e as possibilidades de enfrentá-las. As entrevistas foram gravadas e posteriormente transcritas na integra, procedendo-se à análise qualitativa de conteúdos. Os gestores apresentam percepção da escassa possibilidade de acesso à atenção odontológica nos serviços públicos ofertados em seus municípios e propiciam uma reflexão sobre as principais dificuldades no enfrentamento da equação demanda-efetividade da assistência odontológica.

Palavras-chave Política de saúde, Odontologia, Pesquisa qualitativa 


\section{Introdução}

Os dados sobre acesso a serviços odontológicos no Brasil mostram que um baixo percentual da população refere alguma modalidade de assistência odontológica. De acordo com dados disponíveis pela Pesquisa Nacional por Amostra de Domicílios (PNAD 1998), 20\% da população brasileira referiram nunca ter consultado um cirurgião-dentista. Por outro lado, dados obtidos junto ao DATASUS revelam que, em média, cerca de $10 \%$ da população procura o serviço público de saúde bucal a cada ano, considerando a relação entre exames clínicos de primeira consulta e a população de referência, embora estes dados também devam ser vistos com cautela, em função das deficiências nos nossos sistemas de informação (Roncalli1).

Os indicadores de avaliação no Estado de São Paulo, entre os anos de 1995 e 2000, demonstram que ainda há muito que se trabalhar para que a população tenha acesso à atenção em saúde bucal, uma vez que o acesso à assistência, representado pelo número de primeiras consultas odontológicas pelo total da população multiplicado por 100 , variou de $10,20 \%$ a $11,67 \%$ durante este período (Secretaria de Estado da Saúde 2 ).

Entretanto, Roncalli3 faz uma crítica sobre o modo como esses números são obtidos, pois os dados sobre acesso a serviços de saúde bucal no Brasil em geral possuem "contaminações" e são medidos das mais diversas maneiras, desde a freqüência de consulta nos últimos doze meses à relação entre exames odontológicos na rede pública e a população de referência.

$\mathrm{O}$ inquérito epidemiológico realizado em 2002, no Estado de São Paulo (Secretaria de Estado da Saúde 4 ), revelou que quando perguntados se já haviam ido alguma vez ao cirurgiãodentista (CD), 92\% dos jovens disseram que sim, 91\% dos adultos e $92 \%$ dos idosos também. Destes, 59\% dos jovens visitaram o CD no último ano. Esse percentual cai quanto maior o grupo etário e, nos idosos, a maioria (58\%) visitou o $C D$ há três anos ou mais. Pinto 5 refere que utilização dos serviços odontológicos tem maior freqüência entre os mais jovens e tende a diminuir com a idade.

Sob o ponto de vista epidemiológico, observa-se que, embora o índice CPOD, aos 12 anos de idade, tenha mostrado uma importante redução em anos recentes (Secretaria de Estado da Saúde 4 ), a partir dos 15 anos, o elevado incremento na prevalência de cárie incita a uma reflexão quanto à cobertura prioritariamente oferecida a crianças e a resultante epidemiológica da interrupção desta assistência ao fim do ciclo escolar.

Politicamente, sob a perspectiva da programação, as características e as possibilidades do acesso em cada sistema local de saúde são resultantes de processos históricos, onde atores sociais fazem escolhas racionais e estratégicas que definem regras [...] e assim ordenam a gestão de recursos mobilizados no enfrentamento das complexidades da equação oferta-demanda. Nesta perspectiva, por ser uma construção social, a universalização do acesso à atenção integral em saúde bucal depende de cenários locais específicos (Zanetti6).

Como ponto de partida para o presente estudo, tem-se o dado objetivo que mostra, em um plano mais geral, o baixo acesso da população à assistência odontológica, em contraposição aos princípios de universalização e eqüidade do SUS (Noronha \& Soares 7 ).

Uchimura e Bosi 8 referem que a qualidade em sua dimensão objetiva é mensurável, sendo perfeitamente possivel atribuir valores numéricos a determinados atributos, como tempo de espera ou número de atendimentos. No entanto, o mesmo não ocorre com a dimensão subjetiva (que) habita o espaço das vivências, emoções, sentimentos, não permitindo valorar frustrações por meio de uma linguagem numérica.

A pesquisa qualitativa com entrevistas em profundidade na modalidade depoimento busca aprofundar o sentido social histórico e as representações das pessoas envolvidas no planejamento e formulação de políticas públicas quanto à importância desta questão.

Em função de certo número de propriedades creditadas à percepção, como fornecer o conhecimento do mundo; estar consciente das qualidades sensíveis como cores, sons e formas; e interpretar informações por meio de funções cerebrais superiores (Blackburn 9 ), neste estudo faz-se opção pelo entendimento da percepção como conhecimento consciente do mundo que nos rodeia.

A tomada de consciência que passa do vivido ao pensado não termina o conhecimento, ela faz parte dele (Canguilhen 10, apud Minayo11), de forma que qualquer discurso teórico não é a revelação total da realidade, mas a realização de um real possível ao sujeito, sob condições histórico-sociais dadas (Minayo ${ }^{11}$ ).

Como uma contribuição a esta reflexão, o presente estudo busca analisar como gestores 
municipais percebem o acesso da população à atenção odontológica, na perspectiva do princípio de universalização do SUS.

\section{Método}

O projeto de pesquisa foi aprovado pelo Comitê de Ética em Pesquisa em Seres Humanos da Faculdade de Odontologia de Bauru da Universidade de São Paulo, segundo a Resolução 196/96 do Conselho Nacional de Saúde (Brasil12).

O cenário do estudo corresponde a dois municípios do Estado de São Paulo, pertencentes a regionais de saúde distintas, localizados na região oeste (41.409 habitantes) e região centro-oeste (65.954 habitantes). Os dois municípios, de médio porte, integram o Sistema Único de Saúde (SUS) na forma de "gestão plena da atenção básica" e não são sede de instituições de ensino superior de Odontologia.

Os sujeitos da pesquisa têm como atributo participar da formulação de políticas públicas de saúde e gestão em âmbito local. Foram entrevistados em cada município, respectivamente, 4 profissionais da Secretaria Municipal de Saúde (secretário municipal de saúde, assessor de saúde bucal e dois auxiliares de coordenação de saúde bucal) e 3 profissionais (secretário municipal de saúde, coordenador de saúde bucal e diretor responsável pela área de saúde bucal).

As entrevistas conduzidas por um roteiro semi-estruturado versaram sobre características da assistência odontológica no âmbito público, indicadores de efetividade e opinião do entrevistado sobre as estratégias adotadas pelo município para efetivação do acesso universal. Foram utilizados gravador e fitas-cassete, que posteriormente foram transcritas na íntegra.

A ordenação dos dados permitiu uma abordagem descritiva do material empírico. Foram destacados alguns trechos dos depoimentos que fazem referência aos eixos temáticos, caracterizados como idéias centrais identificadas. Guardando a confidencialidade, a transcrição de algumas expressões é feita a seguir, com objetivo de subsidiar a discussão, utilizando-se letras dispostas aleatoriamente para referenciar as falas dos diferentes sujeitos.

A análise das entrevistas recebeu abordagem qualitativa, buscando estabelecer algumas articulações entre dados e referenciais teóricos (Lefèvre \& Lefèvre13, Minayo11).

\section{Discussão}

A análise de conteúdo discursivo procurou analisar a consonância entre o discurso dos entrevistados e as diretrizes estabelecidas pelo Sistema Único de Saúde, que referem a importância do acesso universal e eqüidade no atendimento e da integralidade das ações de saúde. Em conjunto com os princípios organizativos de regionalização, descentralização, hierarquização e controle social, o "pressuposto" deste estudo é que estas diretrizes, embora conhecidas, não norteiem os serviços desenvolvidos na área de saúde bucal pelas Secretarias Municipais de Saúde.

Este estudo, centrado na análise da dimensão subjetiva da prática, buscou apreender a experiência vivencial de atores sociais que interagem com os programas de saúde bucal em nível local, abordados a partir da ótica do gestor.

A leitura dos depoimentos permitiu a construção de dois eixos de pensamento: na primeira "idéia central", é revelada a preocupação com aspectos da prevenção na prática odontológica, o que tem embasado o planejamento das ações de saúde. Esta concepção é corroborada pela eleição do público-alvo para as ações programáticas de saúde bucal no município, como gestantes e escolares. Para as gestantes, são (realizadas) palestras mais o tratamento curativo. Nas crianças, nós trabalhamos com o tratamento preventivo, curativo e mais a educação $(\mathrm{N})$

No setor público, no início do século 20, houve expressiva redução da assistência odontológica, determinando-se, por esse viés, quais necessidades básicas deveriam ser satisfeitas e restringiu-se esse atendimento aos escolares, na linha do então nascente movimento puericultor e da higiene escolar (Toledo14).

Alguns arranjos de agenda foram descritos, como o agendamento (de) 5 atendimentos programáticos em cada periodo; quando é escolar, é só escolar, mas quando é de comunidade, é a gestante, o idoso, o adolescente e o adulto (J); além disso, os procedimentos coletivos nas escolas (A) e um atendimento básico, nas unidades básicas e em algumas escolas, que é justamente o serviço que a Secretaria de Saúde herdou da Secretaria da Educação (L).

Mais recentemente, bebês e idosos têm encontrado espaço no foco dos gestores, ressaltando a idéia de que todos os grupos teoricamente [grifo das autoras] têm oportunidade de serem atendidos $(\mathrm{J})$. 
Um gestor relata uma possibilidade de ampliação das ações de saúde bucal, com relação ao atendimento ao idoso, (e) à prevenção de câncer bucal (...). Então a gente pode melhorar a qualidade desse atendimento básico e deixar, por exemplo, o atendimento na faculdade, (...) para esse tipo de convênio (A).

Abordando a saúde bucal como fator de exclusão, Botazzo ${ }^{15}$ pondera que ambas não combinam, uma vez que saúde é integralidade. Contudo, na prática odontológica realizada no setor público, existem os pólos de exclusão-inclusão, em que a oferta de serviços de saúde bucal denominada "programática" propicia a exclusão de alguns grupos populacionais aos procedimentos mínimos realizados.

Por outro lado, a inexistência de um planejamento local fica evidenciada nos discursos de gestores, embora a Secretaria Estadual de Saúde do Estado de São Paulo ${ }^{4}$ recomende que a atenção seja baseada em critérios epidemiológicos. Na prática, o que se observa é que os grupos populacionais [...] não são pré-definidos, existe a porta aberta da rede básica, isso na área da assistência individual, lá são atendidos crianças, gestantes, idosos, mas não com uma linha programática pré-definida (A).

Narvai 16 refere que as proposições de prática odontológica da saúde bucal coletiva opõem-se frontalmente à hegemonia da odontologia de mercado. A saúde bucal coletiva tem procurado constituir-se em referência de uma práxis capaz de recuperar para o trabalho em odontologia suas dimensões politica, social, comunitária, preventiva e integral indispensáveis às práticas no campo da saúde que tenham como horizonte sociedades democráticas e solidárias, nas quais as questões de saúde-doença tenham, efetivamente, relevância pública e assim sejam consideradas pelo Estado e pelo conjunto da sociedade.

As diretrizes locais para a área de saúde bucal apontam para a idéia de integralidade, demonstrando que existe alguma iniciativa para pôr em prática o que está na Constituição, não só a assistência, (mas) todas as práticas de saúde bucal que pudessem levar a uma melhoria das condições de saúde bucal da população (J).

$\mathrm{Na}$ segunda "idéia central" identificada a partir dos depoimentos, a ampliação do acesso da população à assistência odontológica surge como uma necessidade, decorrente da percepção empírica que os indicadores de efetividade não sejam satisfatórios.

A falta de mecanismos de acompanhamento, controle e avaliação dos serviços prestados pelos serviços odontológicos municipais vai se evidenciando ao longo dos depoimentos. Hoje (2003), nós temos a avaliação de 2001, nós demos o acesso de 1,9 por habitante, só que a gente percebe que (...) um número bem grande desta população ainda não procura esse serviço (L).

Em função das dificuldades de avaliação de cobertura populacional, restam aos responsáveis pelo planejamento local algumas interrogações. Agora que a gente está definindo mecanismos de avaliação (...) nós procuramos trabalhar um pouco mais o conceito da eficácia. Porque a gente percebeu que mesmo não tendo medido isso adequadamente [...] a gente percebia restauração que cai, re-consulta, enfim, não se resolve o problema. Eu sinto que o acesso ainda é muito restrito $(\mathrm{J})$.

Ao fazer uma reflexão sobre a efetividade (Breilh17), a fila de usuários aguardando pelo atendimento surge como o mais forte indicador presente no cotidiano dos serviços. [...] aqui realmente eu não tenho acesso a esses dados, o que a gente pode avaliar é pela fila. A gente tem que observar principalmente nas unidades básicas a questão da fila e o contato com a própria população (A).

Os depoimentos trazem uma forte impressão da consciência de que é necessário melhorar, aprimorar e ampliar serviços, decorrente de uma prática histórica marcada por baixa eficiência e resolutividade. Eu acho que, de uma maneira geral, toda prefeitura tem pouco recurso, mas de uma certa forma a gente está trabalhando para que todas as pessoas sejam atendidas, não da forma que a gente queria [...], mas [...] a gente está devagar atendendo a todos $(\mathrm{N})$, mas não tem nenhum atendimento de maior complexidade, só extrações e alguns atendimentos básicos (L).

A descrição da assistência prestada nesses municípios reflete um foco predominante na atenção básica, observando-se, contudo, pequenos avanços na oferta de serviços de maior complexidade. Nos adultos, nós fazemos restaurações, extrações, e vamos iniciar o tratamento endodôntico (além da) prótese, dentadura e prótese parcial. Nós temos uma média de 25 pares de dentaduras por mês $(\mathrm{N})$.

A partir do enfoque no desfecho "acesso a serviços", sem que se tome este acesso como significado de melhores condições de saúde, vislumbram-se dificuldades que se antepõem entre os pressupostos constitucionais e sua efetivação. A saúde bucal, em alguns aspectos, acaba sendo relegada sempre a um segundo plano 
quando se fala em políticas de saúde, uma política geral de saúde (A).

Segundo Botazzo ${ }^{18}$, a universalização da assistência à saúde é princípio constitucional e está caracterizado, no âmago do SUS, como elemento constituidor dos direitos do cidadão. No entanto, trata-se de característica que ao mesmo tempo em que expressa desejo coletivo encontra dificuldades em ver-se objetivada, isto é, que se torne efetiva ou se encaminhe como tendência à efetividade. Muitas seriam as causas de tal situação, posto haver concordância, seja na produção científica seja nos relatórios ou na observação, que ainda os serviços públicos não implementaram formas de organização do trabalho que possam garantir acesso pleno e universal aos que deles necessitam.

Quanto ao processo de trabalho, [...] no município, nós temos um problema crônico que é (a) dificuldade de manutenção dos nossos equipamentos odontológicos; são equipamentos muito antigos, nossos colegas cirurgiões-dentistas não trabalham com auxiliares, portanto (...), a efetividade fica difícil de se alcançar com um atendimento com grande eficiência e qualidade para toda a população (M).

Sob o âmbito temático das estratégias necessárias para garantir o acesso universal da população aos serviços odontológicos, os gestores apresentam percepção do acesso escasso e desigual e a necessidade de mecanismos de controle social. Eu acho que a política pública é você dar o acesso à população, mostrar o direito que ele tem e que ele poderia estar junto com a gente (...), porque muitas vezes você percebe que o cidadão não tem conhecimento (...) que ele tem o direito ao acesso (L).

Nesta direção, a reflexão ética deve preceder a formulação de políticas sociais para que sejam justas e considerem todos os seres humanos merecedores de direitos, entendendo a saúde como de inequívoca responsabilidade do Estado (Mendes \& Caldas Júnior ${ }^{19}$ ).

As propostas de políticas públicas em saúde bucal para consolidação do SUS no município passam por [...] uma decisão política de investir na saúde, de reconhecer a saúde como um direito de cidadania. As políticas [...] são no sentido de organizar esse acesso, trabalhar com a população através de colegiados previstos na lei 8.142, [...] nós estamos cumprindo praticamente todos os itens levantados na Conferência Municipal de Saúde (J).

\section{Considerações finais}

Algumas percepções expressas por responsáveis pela elaboração de políticas de saúde demonstram o paradoxo de conhecer princípios importantes que norteiam o SUS, que, contudo, não balizam, na prática, o planejamento das ações programáticas em saúde bucal.

Observa-se que, sob o pretexto de um patrimônio herdado dos programas centrados no atendimento à saúde do escolar, o acesso do "restante" da população à assistência odontológica ocorre de maneira desigual, não organizada e os indicadores empíricos que mostram a baixa efetividade deste sistema são as filas de usuários à espera de atendimento.

A combinação de "uma alta prevalência de doenças com uma lógica de tratamento complexa, demorada e onerosa" (Roncalli ${ }^{3}$ ) permanece um desafio aos gestores públicos de saúde, quando se discute a questão do acesso a serviços.

As inquietações geradas pelo desenvolver da equação demanda-acesso à atenção odontológica remetem à reflexão sobre a necessidade de reordenação da prática odontológica. Importantes contribuições, neste sentido, têm sido desenvolvidas, destacando-se algumas experiências no Brasil, como o modelo de inversão da atenção, o movimento das Cidades Saudáveis, o Programa de Agentes Comunitários de Saúde e o Programa Saúde da Família, entre outros.

Estratégias centradas nos princípios do SUS requerem uma (re)construção, que deve ocorrer no plano coletivo, fundando os alicerces para uma efetiva prática de saúde bucal coletiva. 


\section{Colaboradores}

RB Leal realizou as entrevistas e a transcrição das respostas, além de participar na discussão dos achados. NE Tomita trabalhou na concepção teórica, análise e redação final do texto.

\section{Agradecimentos}

Aos pareceristas ad hoc, pelas contribuições a esta reflexão.

\section{Referências}

1. Roncalli AG. Panorama do acesso à saúde bucal no Brasil. Rev Brasileira de Odontologia e Saúde Coletiva 2002; Suplemento Especial, XVII Enatespo:20.

2. Secretaria de Estado da Saúde (SES-SP). A equipe de saúde bucal no PSF: carga horária do cirurgião-dentista. São Paulo: SES/GATS; 2001.

3. Roncalli AG. A organização da demanda em serviços públicos de saúde bucal: uma contribuição ao estudo da universalidade, da eqüidade e da integralidade em saúde bucal coletiva [Tese] Araçatuba (SP): Faculdade de Odontologia de Araçatuba, Universidade Estadual Paulista Júlio de Mesquita Filho; 2000.

4. Secretaria de Estado da Saúde (SES-SP). Condições de saúde bucal no Estado de São Paulo, em 2000: projeto SB 2000 - condições de saúde bucal da população brasileira no ano 2000 - ampliação da amostra para o Estado de São Paulo. São Paulo: Centro Técnico de Saúde Bucal/SES; 2002.

5. Pinto VG. Saúde bucal coletiva. São Paulo: Editora Santos; 2000.

6. Zanetti CH. Universalização do acesso à atenção integral: um desafio programático ainda não respondido. Rev Brasileira de Odontologia e Saúde Coletiva 2002; Suplemento Especial, XVII Enatespo:20-1.

7. Noronha JC, Soares LT. A política de saúde no Brasil nos anos 90. Rev C S Col 2001; 6(2):445-50.

8. Uchimura KY, Bosi MLM. A polissemia da qualidade na avaliação de programas e serviços de saúde: resgatando a subjetividade. In: Bosi MLM, Mercado FJ, organizadores. Pesquisa qualitativa em serviços de saúde. Rio de Janeiro: Vozes; 2004. p. 75-98.

9. Blackburn S. Dicionário Oxford de Filosofia. Rio de Janeiro: Jorge Zahar Editor; 1997.
10. Canguilhem G. Sobre uma epistemologia concordatária. Epistemologia. Tempo Brasileiro 1972; 28:4756 apud Minayo 1993 p. 250.

11. Minayo MCS. O desafio do conhecimento: pesquisa qualitativa em saúde. 2a ed. São Paulo:Hucitec; Rio de Janeiro: Abrasco; 1993.

12. Brasil. Ministério da Saúde. Conselho Nacional de Saúde. Diretrizes e normas regulamentadoras de pesquisas envolvendo seres humanos. Informe Epidemiológico do SUS 1996; 5(2).

13. Lefèvre F, Lefèvre ANM. O discurso do sujeito coletivo: um novo enfoque em pesquisa qualitativa (desdobramentos). Caxias do Sul: EDUCS; 2003.

14. Toledo JPG. SUS e saúde bucal. Tema desenvolvido no Seminário Internacional ABOPREV: Saúde Bucal para Todos. São Paulo, 29 de abril de 1994.

15. Botazzo C. Saúde como direito de cidadania. Rev Brasileira de Odontologia e Saúde Coletiva 2002. Suplemento Especial, XVII Enatespo: 20.

16. Narvai PC. Odontologia e saúde bucal coletiva. São Paulo: Hucitec; 1994.

17. Breilh J. Epidemiologia: economia, política e saúde. São Paulo: Editora Universidade Estadual Paulista; Fundação para o Desenvolvimento da Unesp; Hucitec; 1991.

18. Botazzo C. A saúde bucal a caminho da universalização: desafios e estratégias. In: VI Encontro Paulista de Administradores e Técnicos do Serviço Público Odontológico (Epatespo) e VI Congresso Paulista de Odontologia em Saúde Coletiva. Sorocaba-SP. 12 October 2002. Disponível em www.saude.sp.gov.br/.

19. Mendes HWB, Caldas Júnior AL. Prática profissional e ética no contexto das políticas de saúde. Rev Lat Am Enfermagem 2001; 9(3):20-6.

Artigo apresentado em 18/06/05 Artigo aprovado em 18/10/2005 Versão final apresentada em 7/11/05 